\title{
Does the consumption of fruits and vegetables differ between Eastern and Western European populations? Systematic review of cross-national studies
}

\author{
Denes Stefler ${ }^{*}$ and Martin Bobak
}

\begin{abstract}
Background: Difference in fruit and vegetable consumption has been suggested as a possible reason for the large gap in cardiovascular disease (CVD) mortality rates between Eastern and Western European populations. However, individual-level dietary data which allow direct comparison across the two regions are rare. In this systematic review we aimed to answer the question whether cross-national studies with comparable individual-level dietary data reveal any systematic differences in fruit and vegetable consumption between populations in Central and Eastern Europe (CEE) and the Former Soviet Union (FSU) compared to Western Europe (WE).

Methods: Studies were identified by electronic search of MEDLINE, EMBASE and Web of Science databases from inception to September 2014, and hand search. Studies which reported data on fruit, vegetable consumption or carotene and vitamin C intake or tissue concentrations of adult participants from both CEE/FSU and WE countries were considered for inclusion. Quality of the included studies was assessed by a modified STROBE statement. Power calculation was performed to determine the statistical significance of the comparison results.

Results: Twenty-two studies fulfilled the inclusion criteria. Fruit consumption was found to be consistently lower in CEE/FSU participants compared to Western Europeans. Results on vegetable intake were less unambiguous. Antioxidant studies indicated lower concentration of beta-carotene in CEE/FSU subjects, but the results for vitamin $\mathrm{C}$ were not consistent.
\end{abstract}

Conclusion: This systematic review suggests that populations in CEE and FSU consume less fruit than Western Europeans. The difference in the consumption of fruit may contribute to the CVD gap between the two regions.

Keywords: Fruit and vegetable consumption, Central and Eastern Europe, Former Soviet Union, Cross-national studies

\section{Background}

Cardiovascular disease (CVD) mortality rates are considerably higher in countries of Central and Eastern Europe (CEE) and Former Soviet Union (FSU) compared Western Europe (WE) [1]. Differences in diet quality between the two regions, fruit and vegetable consumption in particular, has been one of the proposed explanations for this health gap [2-5].

\footnotetext{
* Correspondence: denes.stefler.10@ucl.ac.uk

Department of Epidemiology and Public Health, University College London, 1-19 Torrington Place, London WC1E 6BT, UK
}

The lack of internationally comparable, individuallevel dietary data in Europe is a well-known problem in public health nutrition [6-9]. In 2011, the European Food Safety Authority (EFSA) published the Comprehensive European Food Consumption Database of food consumption data for most EU member states collected by national dietary surveys of individual-level intakes. However, the authors emphasised that due to the differences in data collection methods, the database was not suitable for international comparisons [10]. Other than the differences in dietary assessment methods, the lack of uniform food-grouping and coding system, and differences

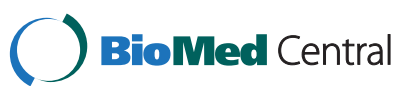


in estimated portion sizes and food composition tables also make the nationally collected and analysed dietary data inadequate for direct country-to-country comparison $[7,8,11]$.

Previous systematic reviews of fruit, vegetable and micronutrient intakes in CEE, FSU and WE countries used data from studies which had been conducted separately in the two regions $[12,13]$. These reviews found that the methodological differences between studies seriously limited the interpretation of the results, and emphasised that the lack of comparable data was especially important in CEE and FSU countries. In this respect, cross-national studies which include participants from both CEE/FSU and WE countries, and collect and analyse dietary data in a standardized way, may be therefore more suitable for direct comparisons of food intakes between the two regions.

The aim of this work was to systematically review cross-national studies which reported individual-level data on consumption of fruits, vegetables, or their indicators, such as vitamin $C$ and carotenoids, of participants from CEE/FSU and WE populations using identical methods for data collection and analysis in the two regions.

\section{Methods}

\section{Search strategy}

MEDLINE, EMBASE and Web of Science databases were searched from inception to September 2014, using search terms described in Appendix 1. References and citation lists of selected papers were studied for additional papers, and hand search of key journals (Public Health Nutrition, European Journal of Clinical Nutrition, European Journal of Public Health) was also performed. No restriction on language was applied.

\section{Inclusion and exclusion criteria}

Original, quantitative, observational epidemiological studies which described fruit, vegetable, antioxidant intakes or antioxidant status of adult participants who live in CEE or FSU countries and provided comparison populations from Western Europe were included in the review. Based on the data collection methods and reported dietary data, the following studies were considered for inclusion: (1) Dietary surveys: studies which reported data on fruit and vegetable intake levels using established nutritional assessment methods such as food frequency questionnaire (FFQ), diet history, dietary record and 24-h diet recall. (2) Health behavioural surveys: reporting data on fruit and vegetable intakes using lifestyle questionnaires with questions regarding fruit or vegetable consumption habits. (3) Antioxidant studies: reporting data on average vitamin $\mathrm{C}$ or carotenoid intakes or status (including plasma, serum and adipose tissue concentrations).
Studies were excluded if data collection methods or the inclusion criteria of participants differed substantially between the two regions. Studies which compared dietary habits between the former East and West Germany were used only if their data collection took place before 1991, because food consumption patterns of East Germans seem to have changed rapidly after the reunification [14].

To avoid bias towards studies which reported more than one exposure of interest from the same participants, we included only one set of data from these studies in the review: data on carotenoid and vitamin $\mathrm{C}$ intake or status were included only if no data on fruit or vegetable consumption were available. If both antioxidant intake and status were reported, only intake data was used, and if data on more than one type of carotenoid concentration were available, only beta-carotene was extracted.

\section{Quality assessment}

Quality of the included studies was assessed by a shortened version of the Strengthening the Reporting of Observational Studies in Epidemiology (STROBE) statement [15]. Modification of the checklist was necessary because several studies described only the nutritional characteristics of the subjects and the analysis of the relationship with disease outcomes was not reported. Therefore four items of the statement, which refer to the variables and outcome results of an analytic study (item nos. 7, 11, 15 and 16), were omitted and the assessment was carried out using the remaining 18 items.

\section{Data analysis}

Most studies described dietary data of participants from more than one country within a certain region. For these studies, the average values for CEE/FSU and WE were calculated and reported in the review.

To take into account the well-documented difference in fruit and vegetable consumption between Northern and Southern European countries [16, 17], both CEE/FSU and WE regions were divided into "south" and "north" subregions (Table 1). If a study reported g/day intake levels of fruits or vegetables of participants from opposite subregions, north/south weighting was applied: the intake figure of the "south" country was multiplied with a weighting factor calculated from FAO data [18] by dividing the average fruit or vegetable supply of all northern countries of that region between 1970 and 2009 by the specific country's average supply over the same time period. For studies reporting data on the percentages of participants eating daily fruits or vegetables, or antioxidant data, no such weighting was carried out because appropriate weighting factors were not available.

If data were collected in winter or spring months in one region and during summer or autumn in the other, seasonal weighting of the CEE/FSU data was applied: the 
Table 1 Grouping of Central and Eastern European (CEE)/former Soviet Union (FSU) and Western European (WE) countries

\begin{tabular}{cll}
\hline Region & Sub-region & Countries \\
\hline CEE/FSU & North & Armenia, Azerbaijan, Belarus, Czech Republic, \\
& & Estonia, Georgia, Hungary, Kazakhstan, \\
& Kyrgyzstan, Latvia, Lithuania, Poland, \\
& Republic of Moldova, Romania, Russian \\
& Federation, Slovakia, Tajikistan, Turkmenistan, \\
& Ukraine, Uzbekistan \\
& Albania, Bosnia and Herzegovina, Bulgaria, \\
& Croatia, Montenegro, Serbia, Slovenia, TFYR \\
& Macedonia \\
WE & Austria, Belgium, Denmark, Finland, France, \\
& Germany, Iceland, Ireland, Liechtenstein, \\
& Luxembourg, Netherlands, Norway, Sweden, \\
& Switzerland, United Kingdom \\
& Andorra, Greece, Italy, Portugal, San Marino, \\
& Spain \\
\hline
\end{tabular}

intake figures were multiplied with a weighting factor which was calculated from the Health Alcohol and Psychosocial Factors in Eastern Europe (HAPIEE) study, which is the largest study in CEE/FSU with dietary data [19]. The weighting factor was determined as the ratio of the energy standardized mean intake level between participants who completed the questionnaire in the summer/autumn months and those who completed it during the winter or spring months. Weighting for seasonal variation was applied only in CEE/FSU because seasonal differences in this region are more substantial than in Western Europe [5, 20, 21].

Most reviewed studies did not report statistical significance of the differences between CEE/FSU and WE. In order to assess whether the reported differences were statistically significant, power calculation was applied. If a study had more than $80 \%$ power to show the described difference as statistically significant on the 0.05 significance level, we considered the reported difference statistically significant. If the power was between $20 \%$ and $80 \%$, we considered that the observed difference was non-significant but the trend was worth noting, and if the power was lower than $20 \%$, the difference was considered negligible. Power calculations were carried out using STATA 12.1 statistical software (StataCorp Texas, USA).

If standard deviation (SD) value was required for power calculation but it was not available from the specific study [22-27], the average SD of fruit, vegetable, vitamin $C$ and beta-carotene intake and concentration levels reported in the European Prospective Investigation into Cancer and Nutrition (EPIC) study cohorts was assumed $[16,28]$. We considered this assumption appropriate because EPIC is the largest international study with such data available and its results suggest that SD values vary in a narrow range irrespectively of study size and mean intake level. In the study which measured adipose tissue beta-carotene concentration [29] the SD reported on a subsample of the same study participants were used [30]. In studies where south/north or seasonal weighting was applied, SD values were multiplied with the same figures as the mean values.

\section{Results}

Characteristics of the reviewed studies

Twenty-two studies met the inclusion criteria: ten dietary surveys [22-26, 31-35], six health behavioural surveys [36-41] and six antioxidant studies [27, 29, 42-45]. Fig. 1 shows the study selection process and Table 2 (see Additional file 1) describes the main features of the included studies. Most studies were cross-sectional in design or reported cross-sectional data from cohort studies. In two studies [29, 32], data were extracted from case-control setting. Participants from $18 \mathrm{CEE} /$ FSU countries and $18 \mathrm{WE}$ states were included in the comparisons and most countries were covered by more than one study. The earliest study [22] reported data from the early 1960s, while the latest data collection took place in 2010 [41]. Sample sizes ranged from 30 to 85921 per region. Five studies [22, 29, 31, 42, 43] recruited only males but the majority gave dietary data for both genders. More than half of the studies applied random sampling method at recruitment and eight $[26,33,37-40,43,45]$ used the general population as the sampling frame.

Overall, the quality of the reviewed studies was good. 15 studies scored 14 or more points on the 18 point scale and only two $[22,44]$ scored less than ten points. Quality of one study [40] was not assessed because it was published as an online database, with no peerreviewed research paper available.

\section{Findings of the reviewed studies}

Table 3 (see Additional file 2) shows the average intake, percentage and concentration values of CEE/FSU and WE participants regarding fruit, vegetable and antioxidants reported by the reviewed studies. The directions of the observed differences and the extent of their significance, determined by power calculation, are also summarised.

Most studies reported their results separately for fruits and vegetables and for males and females. Majority of dietary surveys gave average fruit or vegetable consumption values as mean gram per day intakes, and most of the health behavioural surveys as the percentage of the sample who eat these foods at least once a day.

Regarding fruit intake, both dietary and health behavioural surveys showed consistently lower intakes in CEE/FSU compared to WE. Although six out of nine dietary survey comparisons with adequate power found higher vegetable intake in CEE/FSU countries, the estimates were consistently lower in health behavioural 


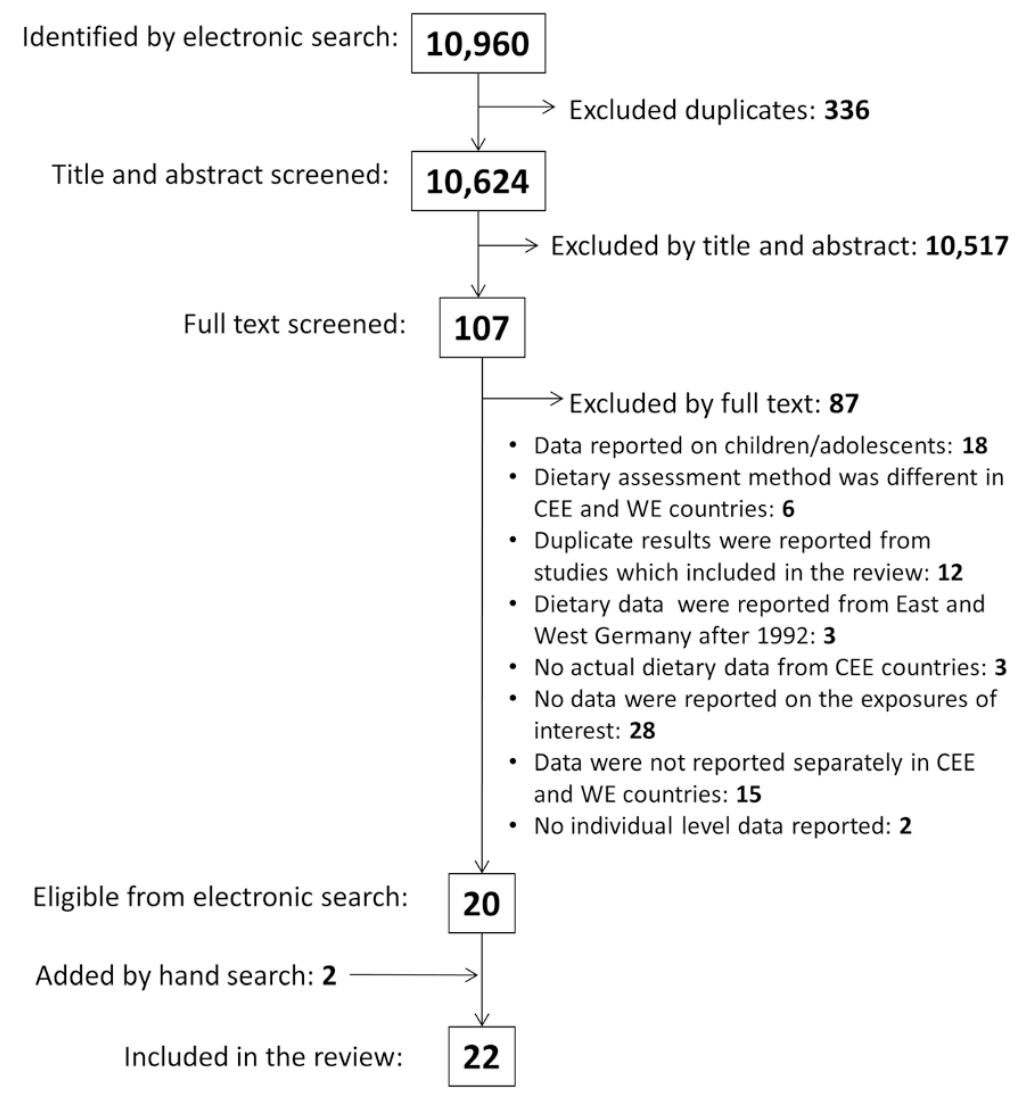

Fig. 1 Flow diagram of the study selection process

surveys. All antioxidant studies indicated lower concentration of beta-carotene in CEE/FSU subjects, but the results for vitamin $\mathrm{C}$ were not consistent. No consistent difference was found between males and females.

\section{Discussion}

This systematic review of cross-national studies on fruit and vegetable intake found consistently lower fruit intake figures in CEE/FSU populations compared to WE, but no consistent difference for vegetable intake between the two regions.

Our results are congruent with ecological dietary data of food availability based on food balance sheets (FBS) and household budgetary surveys (HBS). Comparison of average fruit and vegetable supply in CEE/ FSU and WE countries between 1970 and 2009 suggests clear difference only for fruits but not for vegetables [18]. Similarly, comparison of HBS data from DAFNE database indicates that, on average, the availability of fruits is lower but vegetables is higher in CEE/FSU countries [46].

The inconsistency of our findings regarding vegetable intake can be due to the lack of north/south weighting of health behavioural survey results. For example, in the European Health Interview Survey (EHIS), the largest health behavioural survey included in the review, most participants came from southern countries of Western Europe and northern part of CEE/FSU. If, as a sensitivity analysis, we applied the weighting factors calculated from FAO database for the EHIS results, the comparison showed that the proportion of individuals who consumed vegetables at least once a day was higher in $\mathrm{CEE} / \mathrm{FSU}$ countries, which is similar to most dietary surveys.

On the other hand, most health behaviour surveys had larger sample size than the dietary surveys, and they are also less prone to measurement error. Furthermore, since the main food sources of beta-carotene are vegetables [47], the findings of the antioxidant studies are also in support of the health behavioural survey results and the lower vegetable intake in Eastern Europe.

On the whole, we cannot exclude the possibility that the reason for the inconsistent results regarding vegetable consumption is that there is no actual difference in intake between CEE/FSU and WE populations.

Our review has several limitations. Firstly, it is possible that further published or non-published studies exist 
Table 2 Characteristics of included studies

\begin{tabular}{|c|c|c|c|c|c|c|c|c|c|c|c|c|c|c|}
\hline $\begin{array}{l}1^{\text {st }} \text { author, year } \\
\text { of publication }\end{array}$ & Name of study & $\begin{array}{l}\text { Examined food } \\
\text { or antioxidant }\end{array}$ & $\begin{array}{l}\text { Dietary } \\
\text { assessment }\end{array}$ & $\begin{array}{l}\text { Partic } \\
\text { of ori }\end{array}$ & $\begin{array}{l}\text {-ipants' country } \\
\text { igin }\end{array}$ & $\begin{array}{l}\text { Year of data } \\
\text { collection }\end{array}$ & $\begin{array}{l}\text { Month } \\
\text { of data } \\
\text { collection }\end{array}$ & $\begin{array}{l}\text { Sample } \\
\text { size }\end{array}$ & $\begin{array}{l}\text { Response } \\
\text { rate (\%) }\end{array}$ & $\begin{array}{l}\text { Females } \\
(\%)\end{array}$ & $\begin{array}{l}\text { Age range } \\
\text { or mean } \\
\text { (years) }\end{array}$ & $\begin{array}{l}\text { Sampling } \\
\text { method }\end{array}$ & $\begin{array}{l}\text { Basis of } \\
\text { sample }\end{array}$ & $\begin{array}{l}\text { Quality } \\
\text { score } \\
\text { (max:18) }\end{array}$ \\
\hline \multicolumn{15}{|l|}{ 1. DIETARY SURVEYS } \\
\hline \multirow[t]{2}{*}{ Kromhout 1989 [22] } & $\begin{array}{l}\text { Seven Countries } \\
\text { Study }\end{array}$ & Fruits, vegetables & $7 \mathrm{~d}$ record & CEE: & Yugoslavia & $1960-64$ & Jan-May, Sep & 150 & nd & 0 & $40-59$ & random & $\begin{array}{l}\text { farm/factory } \\
\text { workers, academics }\end{array}$ & 9 \\
\hline & & & & WE: & $\begin{array}{l}\text { Finland, Italy, Greece } \\
\text { Netherlands }\end{array}$ & $1959-65$ & Feb-Sep & 286 & nd & 0 & $40-59$ & random & $\begin{array}{l}\text { village inhabitants, } \\
\text { railroad workers }\end{array}$ & \\
\hline \multirow[t]{2}{*}{ Winkler 1992(31] } & & Fruits, vegetables & $3 \mathrm{~d}$ record & CEE: & GDR & 1987 & Oct-Dec & 132 & 73 & 0 & $45-64$ & random & urban inhabitants & 11 \\
\hline & & & & WE: & FDR & $1984-85$ & Oct-May & 424 & 70 & 0 & $45-64$ & cluster & urban inhabitants & \\
\hline \multirow[t]{2}{*}{ Schroll 1996 [23] } & SENECA & Fruits, vegetables & Diet history & CEE: & Poland & 1993 & Jan-Jun & 120 & $51+$ & 61 & $74-79$ & random & urban inhabitants & 13 \\
\hline & & & & WE: & $\begin{array}{l}\text { Belgium, Denmark, } \\
\text { France, Italy, } \\
\text { Netherlands, Portugal, } \\
\text { Spain, UK, Switzerland }\end{array}$ & 1993 & Jan-Jun & 1237 & $51+$ & 51 & $74-79$ & random & urban inhabitants & \\
\hline \multirow{2}{*}{$\begin{array}{l}\text { Karamanos } \\
2002[24]\end{array}$} & & Fruits, vegetables & Diet history & CEE: & Bulgaria & nd & nd & 288 & nd & 50 & $35-60$ & random & urban inhabitants & 14 \\
\hline & & & & WE: & Italy, Greece & nd & nd & 1058 & nd & 54 & $35-60$ & random & $\begin{array}{l}\text { urban and rural } \\
\text { inhabitants }\end{array}$ & \\
\hline \multirow{2}{*}{$\begin{array}{l}\text { Serra-Majem } \\
2003[25]\end{array}$} & WHO-CINDI & Fruits, vegetables & $24 \mathrm{hr}$ recall & CEE: & Poland & $1991-94$ & nd & 4440 & nd & 50 & $20-65$ & random & factory workers & 14 \\
\hline & & & & WE: & Spain & 1992 & nd & 2757 & 69 & nd & $6-75$ & random & general population & \\
\hline \multirow{2}{*}{$\begin{array}{l}\text { Petkeviciene } \\
2009 \text { [26] }\end{array}$} & NORBAGREEN & Fruits, vegetables & FFQ & CEE: & Lithuania & 2002 & Apr & 99 & 68 & 57 & $19-75$ & random & general population & 15 \\
\hline & & & & WE: & Finland & 2002 & Jan-May & 125 & 91 & nd & $25-64$ & random & general population & \\
\hline \multirow[t]{2}{*}{ Lixandru 2010 [32] } & & Fruits, vegetables & FFQ & CEE: & Romania & 2005 & Apr-Nov & 40 & nd & 30 & 63 & convenience & diabetic patients & 12 \\
\hline & & & & WE: & Belgium & 2005 & Apr-Nov & 30 & nd & 20 & 62 & convenience & diabetic patients & \\
\hline \multirow[t]{2}{*}{ Paalanen 2011 [33] } & & Fruits, vegetables & FFQ & CEE: & Russia & $1992-07$ & Mar-May & 2672 & $45-92$ & 57 & $25-64$ & random & general population & 16 \\
\hline & & & & WE: & Finland & 1992-02 & Mar-May & 4365 & $67-81$ & 53 & $25-64$ & random & general population & \\
\hline \multirow[t]{2}{*}{ Crispim 2011 [34] } & EFCOVAL & Fruits, vegetables & $24 \mathrm{hr}$ recall & CEE: & Czech Republic & $2007-08$ & Oct-Apr & 118 & nd & 51 & $45-65$ & convenience & healthy individuals & 16 \\
\hline & & & & WE: & $\begin{array}{l}\text { Belgium, France, } \\
\text { Norway Netherlands, }\end{array}$ & $2007-08$ & $\begin{array}{l}\text { Apr-Jul, Oct- } \\
\text { Apr }\end{array}$ & 482 & nd & 50 & $45-65$ & convenience & healthy individuals & \\
\hline \multirow[t]{2}{*}{ El Ansari 2012 [35] } & CNSHS & Fruits, vegetables & FFQ & CEE: & Bulgaria, Poland & 2005 & nd & 1143 & 95 & 70 & 21 & convenience & university students & 14 \\
\hline & & & & WE: & Denmark, Germany & 2005 & nd & 1236 & $85-92$ & 53 & 21 & convenience & university students & \\
\hline \multicolumn{15}{|c|}{$\begin{array}{l}\text { 2. HEALTH BEHAVIOUR } \\
\text { SURVEYS }\end{array}$} \\
\hline Wardle 1997 [36] & EHBS & Fruits & na & CEE: & $\begin{array}{l}\text { Poland, Hungary, } \\
\text { GDR }\end{array}$ & $1989-92$ & nd & 2293 & $90-100$ & 51 & 22 & convenience & university students & 13 \\
\hline
\end{tabular}


Table $\mathbf{2}$ Characteristics of included studies (Continued)

\begin{tabular}{|c|c|c|c|c|c|c|c|c|c|c|c|c|c|c|}
\hline & & & & WE: & $\begin{array}{l}\text { Austria, Belgium, FDR, } \\
\text { UK Denmark, Finland, } \\
\text { Spain, France, Greece, } \\
\text { Iceland, Ireland, Italy, } \\
\text { Sweden, Netherlands, } \\
\text { Norway, Portugal, } \\
\text { Switzerland }\end{array}$ & $1989-92$ & nd & 14192 & $90-100$ & 56 & 21 & convenience & university students & \\
\hline \multirow[t]{2}{*}{ Prattala 2007 [37] } & $\begin{array}{l}\text { Finbalt Health } \\
\text { Monitor project }\end{array}$ & Fruits & na & CEE: & $\begin{array}{l}\text { Estonia, Latvia, } \\
\text { Lithuania }\end{array}$ & 1998-02 & Apr-May & 15740 & $62-80$ & 57 & $20-64$ & random & general population & 16 \\
\hline & & & & WE: & Finland & 1998-02 & Apr-May & 9354 & $65-70$ & 53 & $20-64$ & random & general population & \\
\hline \multirow[t]{2}{*}{ Prattala 2009 [38] } & EUROTHIENE & Vegetables & na & CEE: & $\begin{array}{l}\text { Estonia, Latvia, } \\
\text { Lithuania }\end{array}$ & 2000-04 & nd & 14219 & $60-73$ & 58 & $20-64$ & random & general population & 15 \\
\hline & & & & WE: & $\begin{array}{l}\text { Finland, Denmark, } \\
\text { Spain, Germany, } \\
\text { France, Italy }\end{array}$ & 1998-04 & nd & 86924 & $61-87$ & 51 & $20-64$ & random & general population & \\
\hline \multirow[t]{2}{*}{ Hall 2009 [39] } & WHS & Fruits, vegetables & na & CEE: & $\begin{array}{l}\text { Bosnia and } \\
\text { Herzegovina, Croatia, } \\
\text { Czech Republic, } \\
\text { Estonia, Georgia, } \\
\text { Hungary, Kazakhstan, } \\
\text { Latvia, Russia, Slovakia, } \\
\text { Slovenia, Ukraine }\end{array}$ & 2002-03 & nd & 22475 & $69-100$ & 53 & $18-99$ & random & general population & 15 \\
\hline & & & & WE: & Spain & 2002-03 & nd & 5448 & 86 & 60 & $18-99$ & random & general population & \\
\hline \multirow[t]{2}{*}{$\begin{array}{l}\text { European } \\
\text { Commission } \\
2013 \text { [40] }\end{array}$} & EHIS & Fruits, vegetables & na & CEE: & $\begin{array}{l}\text { Bulgaria, Czech } \\
\text { Republic, Estonia, } \\
\text { Latvia, Hungary, } \\
\text { Poland, Romania, } \\
\text { Slovakia, Slovenia }\end{array}$ & 2006-09 & nd & 85921 & $56-89$ & 53 & $15-99$ & random & general population & na \\
\hline & & & & WE: & $\begin{array}{l}\text { Belgium, Greece, } \\
\text { Spain, France }\end{array}$ & 2006-09 & nd & 62700 & $60-96$ & 55 & $15-99$ & random & general population & \\
\hline \multirow[t]{2}{*}{ Burisch 2014 [41] } & ECCO-EpiCom & Fruits, Vegetables & na & CEE: & $\begin{array}{l}\text { Croatia, Czech Rep, } \\
\text { Estonia, Hungary, } \\
\text { Lithuania, Moldova, } \\
\text { Romania, Russia }\end{array}$ & 2010 & Jan-Dec & 249 & $76+$ & 42 & $15+$ & convenience & $\begin{array}{l}\text { IBD patients } \\
\text { (at diagnosis) }\end{array}$ & 16 \\
\hline & & & & WE: & $\begin{array}{l}\text { Cyprus, Denmark, } \\
\text { Finland, Greece, } \\
\text { Iceland, Ireland, } \\
\text { Israel, Italy, Portugal, } \\
\text { Spain, Sweden, UK }\end{array}$ & 2010 & Jan-Dec & 933 & $76+$ & 46 & $15+$ & convenience & $\begin{array}{l}\text { IBD patients } \\
\text { (at diagnosis) }\end{array}$ & \\
\hline \multicolumn{15}{|l|}{$\begin{array}{l}\text { 3. ANTIOXIDANT } \\
\text { STUDIES }\end{array}$} \\
\hline \multirow[t]{2}{*}{ Kardinaal 1993 [29] } & EURAMIC & $\begin{array}{l}\text { Beta-carotene in } \\
\text { adipose tissue }\end{array}$ & na & CEE: & Russia & $1991-92$ & nd & 200 & $79-97$ & 0 & 51 & convenience & $\begin{array}{l}\text { hospital patients, } \\
\text { healthy controls }\end{array}$ & 16 \\
\hline & & & & WE: & $\begin{array}{l}\text { Finland, Germany, } \\
\text { Netherlands, Norway, } \\
\text { UK, Spain, Switzerland }\end{array}$ & $1991-92$ & nd & 1180 & $50-98$ & 0 & 54 & convenience & $\begin{array}{l}\text { hospital patients, } \\
\text { healthy controls }\end{array}$ & \\
\hline
\end{tabular}


Table 2 Characteristics of included studies (Continued)

\begin{tabular}{|c|c|c|c|c|c|c|c|c|c|c|c|c|c|c|}
\hline \multirow[t]{2}{*}{ Kristenson 1997 [42] } & LiVicordia & $\begin{array}{l}\text { Beta-carotene in } \\
\text { plasma }\end{array}$ & na & CEE: & Lithuania & $1993-94$ & Oct-Jun & 100 & 83 & 0 & 50 & random & urban inhabitants & 14 \\
\hline & & & & WE: & Sweden & 1993-94 & Oct-Jun & 95 & 83 & 0 & 50 & random & urban inhabitants & \\
\hline \multirow[t]{2}{*}{ Bobak 1998 [27] } & & $\begin{array}{l}\text { Beta-carotene in } \\
\text { plasma }\end{array}$ & na & CEE: & Czech Republic & 1992 & Sep-Nov & 136 & 70 & 49 & $40-59$ & random & urban inhabitants & 14 \\
\hline & & & & WE: & UK & $1991-93$ & nd & 358 & 73 & 31 & $40-59$ & random & civil servants & \\
\hline \multirow[t]{2}{*}{ Bobak 1999 [43] } & & $\begin{array}{l}\text { Beta-carotene in } \\
\text { plasma }\end{array}$ & na & CEE: & Czech Republic & 1995 & Apr-Jun & 188 & 70 & 0 & $45-64$ & random & general population & 17 \\
\hline & & & & WE: & Germany & 1995 & Apr-Jun & 153 & 70 & 0 & $45-64$ & random & general population & \\
\hline \multirow[t]{2}{*}{ Miere 2007 [44] } & & Vitamin C intake & $24 \mathrm{~h}$ recall & CEE: & Romania & nd & nd & 312 & nd & 87 & 21 & convenience & university students & 8 \\
\hline & & & & WE: & Spain & nd & nd & 918 & nd & 58 & 22 & convenience & university students & \\
\hline \multirow[t]{2}{*}{ Woodside 2013 [45] } & EUREYE & $\begin{array}{l}\text { Vitamin } C \text { and } \\
\text { Beta-carotene } \\
\text { in plasma }\end{array}$ & na & CEE: & Estonia & 2000-03 & nd & 833 & 58.6 & 66 & $65+$ & random & general population & 15 \\
\hline & & & & WE: & $\begin{array}{l}\text { Norway, UK, France, } \\
\text { Italy, Greece, Spain }\end{array}$ & $2000-03$ & nd & 3300 & $36-56$ & 52 & $65+$ & random & general population & \\
\hline
\end{tabular}

WHO-CINDI, World Health Organization Countrywide Integrated Non-communicable Disease Intervention; NORBAGREE, Consumption of vegetables and fruits and other dietary health indicator foods in the Nordic and Baltic countries; EFCOVAL, European Food Consumption Validation; CNSHS, Cross National Student Health Survey; EHBS, European Health and Behaviour Survey; WHS, World Health Survey; EHIS, European Health Baltic countries; EFCOVAL, European Food Consumption Validation; CNSHS, Cross National Student Health Survey; EHBS, European Health and Behaviour Survey; WHS, World Health Survey; EHIS, European Health
Interview Survey; EURAMIC, European Community Multicentre Study on Antioxidants, Myocardial Infarction and Breast Cancer; LiVicordia, Linkoping-Vilnius Coronary Disease Risk Assessment Study; ECCO-EpiCom, European Crohn's and Colitis Organization's Epidemiological Committee study; FDR, Federal Republic of Germany; GDR, German Democratic Republic; CEE: Central and Eastern Europe (or Former Soviet Union); WE, Western Europe; FFQ, Food frequency questionnaire; na, not applicable; nd, no data available; IBD, Inflammatory bowel disease

Western Europe; $\mathrm{FFQ}$, Food frequency questionnaire; na, not applicable; nd, no dased
*Based on evaluation using a modified STROBE checklist; †Overall response rate 
Table 3 Summary results of the included studies

\begin{tabular}{|c|c|c|c|c|c|c|c|c|c|c|}
\hline \multirow{2}{*}{$\begin{array}{l}1^{\text {st }} \text { author, year } \\
\text { of publication }\end{array}$} & \multirow{2}{*}{$\begin{array}{l}\text { Unit of } \\
\text { measurement }\end{array}$} & \multirow[t]{2}{*}{ Sex } & \multicolumn{3}{|l|}{ CEE countries } & \multicolumn{3}{|l|}{ WE countries } & \multirow[t]{2}{*}{ Power } & \multirow{2}{*}{$\begin{array}{l}\text { Summary: CEE } \\
\text { compared to WE }\end{array}$} \\
\hline & & & $\begin{array}{l}\text { Average intake, } \\
\text { cc. or \% }\end{array}$ & Range* & SD & $\begin{array}{l}\text { Average intake, } \\
\text { cc. or } \%\end{array}$ & Range* & SD & & \\
\hline \multicolumn{11}{|l|}{ 1. DIETARY SURVEYS } \\
\hline \multicolumn{11}{|l|}{ FRUITS } \\
\hline Kromhout 1989 [22]§ | & g/day intake & M & 58.6 & $1.0-153.6$ & $207.3+$ & 132.1 & 21.3-310.9 & $178.3+$ & 0.96 & LOWER \\
\hline Winkler 1992 [31] & $\mathrm{g} /$ day intake & M & 98.0 & & 145.3 & 101.0 & & 164.3 & 0.05 & no difference \\
\hline \multirow[t]{2}{*}{ Schroll 1996 [23]§ } & $\mathrm{g} /$ day intake & M & 186.0 & & $239.1+$ & 234.0 & $120.0-532.5$ & $230.2+$ & 0.26 & lower-ns \\
\hline & & $\mathrm{F}$ & 162.0 & & $210.2+$ & 208.0 & $135.0-399.6$ & $202.4 \dagger$ & 0.43 & lower-ns \\
\hline \multirow[t]{2}{*}{ Karamanos 2002 [24] } & $\mathrm{g} /$ day intake & M & 293.0 & & $239.1+$ & 315.0 & $236.0-355.0$ & $239.1+$ & 0.16 & no difference \\
\hline & & $\mathrm{F}$ & 303.0 & & $210.2+$ & 325.7 & $234.0-377.0$ & $210.2+$ & 0.21 & lower-ns \\
\hline Serra-Majem 2003 [25]§ & $\mathrm{g} /$ day intake & $M+F$ & 137.0 & & $224.7+$ & 290.0 & & $218.0+$ & 1.00 & LOWER \\
\hline Petkeviciene 2009 [26] & $\mathrm{p} /$ month intake & $M+F$ & 20.8 & & $84.3+$ & 29.4 & & $84.3+$ & 0.12 & no difference \\
\hline \multirow[t]{2}{*}{ Lixandru 2010 [3] } & $\%$ eat daily & M & 100.0 & & na & 89.5 & & na & 0.34 & higher-ns \\
\hline & & $\mathrm{F}$ & 100.0 & & na & 100.0 & & na & na & no difference \\
\hline \multirow[t]{2}{*}{ Paalanen 2011 [33] } & $\%$ eat daily & M & 14.0 & $2.0-31.0$ & na & 52.3 & $43.0-61.0$ & na & 1.00 & LOWER \\
\hline & & $\mathrm{F}$ & 26.0 & $4.0-50.0$ & na & 73.3 & $66.0-82.0$ & na & 1.00 & LOWER \\
\hline \multirow[t]{2}{*}{ Crispim 2011 [34] } & $\mathrm{g} /$ day intake & M & 207.0 & & 176.7 & 197.0 & $163.0-228.0$ & 175.1 & 0.07 & no difference \\
\hline & & F & 226.0 & & 155.7 & 230.5 & $194.0-265.0$ & 151.1 & 0.05 & no difference \\
\hline \multirow[t]{2}{*}{ El Ansari 2012 [35] } & $\%$ eat daily & M & 31.6 & $23.8-39.4$ & na & 30.4 & $28.6-32.1$ & na & 0.05 & no difference \\
\hline & & F & 46.8 & $39.5-54.1$ & na & 51.6 & $47.8-55.4$ & na & 0.42 & lower-ns \\
\hline \multicolumn{11}{|l|}{ VEGETABLES } \\
\hline Kromhout 1989 [22]§ | & $\mathrm{g} /$ day intake & M & 240.0 & $159.0-276.0$ & $198.2+$ & 102.6 & $57.3-227$ & $88.1+$ & 1.00 & HIGHER \\
\hline Winkler 1992 [31] & $\mathrm{g} /$ day intake & M & 126.0 & & 154.8 & 124.0 & & 154.8 & 0.05 & no difference \\
\hline \multirow[t]{2}{*}{ Schroll 1996 [23]§ } & g/day intake & M & 341.0 & & $154.8+$ & 288.0 & $82.4-461.0$ & $128.1+$ & 0.63 & higher-ns \\
\hline & & F & 297.0 & & $143.9+$ & 238.0 & $77.0-383.0$ & $121.0+$ & 0.92 & HIGHER \\
\hline \multirow[t]{2}{*}{ Karamanos 2002 [24] } & $\mathrm{g} /$ day intake & M & 243.0 & & $154.8+$ & 189.0 & $168.0-214.0$ & $154.8+$ & 0.96 & HIGHER \\
\hline & & $\mathrm{F}$ & 291.0 & & $143.9+$ & 197.3 & $178.0-222.0$ & $143.9+$ & 1.00 & HIGHER \\
\hline Serra-Majem 2003 [25]§ & $\mathrm{g} /$ day intake & $\mathrm{M}+\mathrm{F}$ & 288.0 & & $149.4 \dagger$ & 97.1 & & $68.7 \dagger$ & 1.00 & HIGHER \\
\hline Petkeviciene 2009 [26] & $\mathrm{p} / \mathrm{month}$ intake & $\mathrm{M}+\mathrm{F}$ & 29.9 & & $56.0+$ & 29.1 & & $56.0+$ & 0.05 & no difference \\
\hline \multirow[t]{2}{*}{ Lixandru 2010 [32] } & $\mathrm{g} /$ day intake & M & 287.0 & & 189.4 & 269.9 & & 108.1 & 0.07 & no difference \\
\hline & & $\mathrm{F}$ & 258.3 & & 157.9 & 283.3 & & 125.2 & 0.06 & no difference \\
\hline \multirow[t]{2}{*}{ Paalanen 2011 [33] } & $\%$ eat daily & M & 15.0 & $10.0-24.0$ & na & 48.7 & $44.0-54.0$ & na & 1.00 & LOWER \\
\hline & & $\mathrm{F}$ & 22.3 & $11.0-35.0$ & na & 70.7 & $69.0-72.0$ & na & 1.00 & LOWER \\
\hline
\end{tabular}


Table 3 Summary results of the included studies (Continued)

\begin{tabular}{|c|c|c|c|c|c|c|c|c|c|c|}
\hline \multirow[t]{2}{*}{ Crispim 2011 [34] } & $\mathrm{g} /$ day intake & M & 162.0 & & 121.1 & 201.0 & $168.0-222.0$ & 112.8 & 0.60 & lower-ns \\
\hline & & $\mathrm{F}$ & 157.0 & & 99.1 & 202.3 & $166.0-254.0$ & 108.5 & 0.87 & LOWER \\
\hline \multirow[t]{2}{*}{ El Ansari 2012 [35] } & $\%$ eat daily & M & 37.8 & $23.9-51.6$ & na & 24.4 & $23.3-25.4$ & na & 0.99 & HIGHER \\
\hline & & $\mathrm{F}$ & 44.9 & $28.0-61.8$ & na & 42.0 & $37.5-46.4$ & na & 0.18 & no difference \\
\hline \multicolumn{11}{|c|}{ 2. HEALTH BEHAVIOUR SURVEYS } \\
\hline \multicolumn{11}{|l|}{ FRUITS } \\
\hline \multirow[t]{2}{*}{ Wardle 1997 [36] } & $\%$ eat daily & M & 40.0 & $36.0-45.0$ & na & 42.9 & $23.0-78.0$ & na & 0.43 & lower-ns \\
\hline & & $\mathrm{F}$ & 65.0 & $59.0-74.0$ & na & 61.1 & $36.2-86.0$ & na & 0.72 & higher-ns \\
\hline \multirow[t]{2}{*}{ Prattala 2007 [37] } & $\%$ eat daily & M & 11.0 & $10.0-12.0$ & na & 18.0 & & na & 1.00 & LOWER \\
\hline & & $\mathrm{F}$ & 20.3 & $17.0-25.0$ & na & 36.0 & & na & 1.00 & LOWER \\
\hline \multirow[t]{2}{*}{ EHIS 2013 [40] } & $\%$ eat daily & M & 52.8 & $39.4-66.8$ & na & 60.6 & $57.9-66.0$ & na & 1.00 & LOWER \\
\hline & & $\mathrm{F}$ & 67.0 & $49.2-82.3$ & na & 69.1 & $62.3-74.5$ & na & 1.00 & LOWER \\
\hline Burisch 2014[41] & $\%$ eat daily & $M+F$ & 43.4 & & na & 54.3 & & na & 0.87 & LOWER \\
\hline \multicolumn{11}{|l|}{ VEGETABLES } \\
\hline \multirow[t]{2}{*}{ Prattala 2009 [38] } & $\%$ eat daily & M & 22.5 & $16.1-27.5$ & na & 32.1 & 24.7-39.1 & na & 1.00 & LOWER \\
\hline & & $\mathrm{F}$ & 30.4 & $25.0-33.4$ & na & 45.9 & $36.9-59.1$ & na & 1.00 & LOWER \\
\hline \multirow[t]{2}{*}{ EHIS 2013 [40] } & $\%$ eat daily & M & 54.8 & $44.2-71.3$ & na & 68.6 & $56.0-82.7$ & na & 1.00 & LOWER \\
\hline & & $\mathrm{F}$ & 62.5 & $55.0-78.6$ & na & 74.2 & $65.3-87.4$ & na & 1.00 & LOWER \\
\hline Burisch 2014 [41] & $\%$ eat daily & $\mathrm{M}+\mathrm{F}$ & 49.0 & & na & 60.1 & & na & 0.88 & LOWER \\
\hline \multicolumn{11}{|c|}{ FRUITS AND VEGETABLES } \\
\hline \multirow[t]{2}{*}{ Hall 2009 [3] } & $\%$ eat $>=5 \mathrm{p} /$ day & M & 18.1 & $8.0-44.5$ & na & 22.0 & & na & 0.98 & LOWER \\
\hline & & $\mathrm{F}$ & 23.5 & $9.4-49.7$ & na & 24.9 & & na & 0.38 & lower-ns \\
\hline \multicolumn{11}{|c|}{ 3. ANTIOXIDANT STUDIES } \\
\hline \multicolumn{11}{|l|}{ BETA CAROTENE } \\
\hline Kardinaal 1993 [29] & ug/g fatty acid & M & 0.51 & $0.45-0.56$ & 0.80 & 0.42 & $0.18-0.59$ & 0.80 & 0.31 & higher-ns \\
\hline Kristenson 1997 [42] & umol// cc. & M & 0.38 & & 0.20 & 0.51 & & 0.32 & 0.92 & LOWER \\
\hline \multirow[t]{2}{*}{ Bobak 1998 [27] } & umol/l cc. & M & 0.39 & & $0.26+$ & 0.77 & & $0.26+$ & 1.00 & LOWER \\
\hline & & $\mathrm{F}$ & 0.52 & & $0.40+$ & 0.97 & & $0.40+$ & 1.00 & LOWER \\
\hline Bobak 1999 [43] & umol/l cc.** & M & 0.11 & & 0.08 & 0.20 & & 0.21 & 1.00 & LOWER \\
\hline \multirow[t]{2}{*}{ Woodside 2013 [45] } & umol/l cc & M & 0.25 & & 0.26 & 0.34 & $0.19-0.48$ & 0.31 & 1.00 & LOWER \\
\hline & & $\mathrm{F}$ & 0.36 & & 0.34 & 0.44 & $0.30-0.67$ & 0.37 & 1.00 & LOWER \\
\hline \multicolumn{11}{|l|}{ VITAMIN C } \\
\hline Miere 2007 [44] & mg/day intake & M & 80.3 & & 54.8 & 106.2 & & 83.4 & 0.77 & lower-ns \\
\hline
\end{tabular}


Table 3 Summary results of the included studies (Continued)

\begin{tabular}{|c|c|c|c|c|c|c|c|c|c|}
\hline & & $\bar{F}$ & 88.8 & 67.9 & 124.4 & & 94.8 & 1.00 & LOWER \\
\hline \multirow[t]{2}{*}{ Woodside 2013 [45] } & umol// cc. & M & 42.0 & 23.8 & 38.0 & $32.7-44.4$ & 23.1 & 0.74 & higher-ns \\
\hline & & $\mathrm{F}$ & 54.5 & 27.7 & 48.5 & $43.5-52.4$ & 23.4 & 1.00 & HIGHER \\
\hline
\end{tabular}

M, Males; F, Females; p, portion; EHIS, European Health Interview Survey; na, not applicable; cc., concentration

* Range of intake levels, percentages or concentrations if data was reported from more than one country or site

TSD assumed from EPIC study

fLOWER: Intake level, percentage or concentration significantly lower in CEE/FSU countries compared to data from WE, (power $>0.80$ ); HIGHER: Intake level, percentage or concentration significantly higher in

$\mathrm{CEE} / \mathrm{FSU}$ countries compared to data from WE, (power $>0.80$ ); lower-ns: Intake level, percentage or concentration lower in CEE/FSU but difference not significant (power $<0.80$ and $>0.20$ ); higher-ns: Intake level, percentage or concentration higher in CEE/FSU but difference not significant (power $<0.80$ and $>0.20$ ); no difference: power $<0.20$

§:North-south weighting was applie

I:Seasonal weighting was applied

**Calculated from reported data using molar mass $=537 \mathrm{~g}$ 
which we did not identify during the search. However, cross-national studies tend to require substantial funding, logistics and international cooperation between institutions, which often go hand in hand with the endeavour to publish the work in internationally reputable journals which can be found in the electronic databases we searched. In addition, as we applied no language restriction in the electronic search, the possibility of finding studies from non-English speaking countries was increased.

Secondly, our data analysis involved several assumptions. The weighting factors from FAO database and HAPIEE study were the best options currently available for these purposes, and the SD values brought over from EPIC study did not influence the direction of the results, it only helped to decide whether the studies were sufficiently large to draw meaningful conclusions of their findings.

Although the reviewed studies included participants from a large number of CEE/FSU and WE countries, some of them providing nationally representative food consumption data, specific comparisons were representative only for a small proportion of the whole CEE/FSU and WE populations. Because large differences exist in fruit and vegetable intakes within the regions, the reported comparisons can only be seen as pixels of a much larger picture. The complete picture will emerge only when nationally representative, comparable dietary data is available for most European countries; in fact, this is the main aim of EFSA's on-going "EU Menu" project [48].

\section{Conclusion}

This systematic review supports previous data that people in CEE/FSU countries consume less fruit than Western Europeans, and that the difference in vegetable intake is probably less clear-cut. Since inadequate consumption of fruit is suggested as a modifiable risk factor for CVD $[49,50]$, the difference in fruit intake may contribute to the gap in CVD mortality rates between the two regions.

\section{Additional files}

Additional file 1: Characteristics of included studies.

Additional file 2: Summary of results of included studies.

Additional file 3: Search terms used for MEDLINE search.

\footnotetext{
Abbreviations

CV: Cardiovascular disease; CEE: Central and Eastern Europe; DAFNE: Data Food Networking; EFSA: European Food Safety Authority; EHIS: European Health Interview Survey; EPIC: European Prospective Investigation into Cancer and Nutrition study; FAO: Food and Agriculture Organization; FBS: Food balance sheet; FFQ: Food frequency questionnaire; FSU: Former Soviet Union; HAPIEE: Health Alcohol and Psychosocial Factors in Eastern Europe study; HBS: Household budgetary Survey; STROBE: Strengthening the Reporting of Observational Studies in Epidemiology; WE: Western Europe.
}

\section{Competing interests}

The authors declare that they have no competing interests.

\section{Authors' contributions}

DS carried out the literature search, quality assessment of reviewed studies and the data analysis. DS and MB wrote the manuscript. Both authors read and approved the final manuscript

\section{Acknowledgements}

The work was partly supported by the Russian Scientific Foundation (\# 14-45-00030). D Stefler was supported by the British Heart Foundation.

Received: 24 November 2014 Accepted: 5 May 2015

Published: 15 June 2015

\section{References}

1. WHO Regional Office for Europe (2014). Health for All Database. http:// data.euro.who.int/hfadb/

2. Ginter E. Cardiovascular risk factors in the former communist countries. Analysis of 40 European MONICA populations. Eur J Epidemiol. 1995;11:199-205.

3. Pomerleau J, McKee M, Lobstein T, Knai C. The burden of disease attributable to nutrition in Europe. Public Health Nutr. 2003;6:453-61.

4. Powles JW, Zatonski W, Vander Hoorn S, Ezzati M. The contribution of leading diseases and risk factors to excess losses of healthy life in Eastern Europe: burden of disease study. BMC Public Health. 2005;5:116.

5. Zatonski WA. Epidemiological analysis of health situation development in Europe and its causes until 1990. Ann Agric Environ Med. 2011;18:194-202.

6. West CE. Eurofoods: towards compatibility of nutrient data banks in Europe. Ann Nutr Metab. 1985;29 Suppl 1:1-72.

7. de Boer EJ, Slimani N, Boeing H, Feinberg M, Leclercq C, Trolle E, et al. Rationale and methods of the European Food Consumption Validation (EFCOVAL) Project. Eur J Clin Nutr. 2011;65 Suppl 1:S1-4.

8. Charrondiere UR, Vignat J, Moller A, Ireland J, Becker W, Church S, et al. The European Nutrient Database (ENDB) for Nutritional Epidemiology. J Food Compost Anal. 2002;15:435-51.

9. WHO Regional Office for Europe (2010): Report of the Workshop on integration of data on household food availability and individual dietary intakes. Copenhagen. 1-39

10. European Food Safety Authority. Use of the EFSA Comprehensive European Food Consumption Database in Exposure Assessment. EFSA J. 2011;9:2097.

11. Ireland J, van Erp-Baart AM, Charrondiere UR, Møller A, Smithers G, Trichopoulou A, et al. Selection of a food classification system and a food composition database for future food consumption surveys. Eur J Clin Nutr. 2002;56 Suppl 2:S33-45.

12. Lesser S, Pauly L, Volkert D, Stehle P, Ageing Nutrition Group. Nutritional situation of the elderly in Eastern/Baltic and Central/Western Europe - the AgeingNutrition project. Ann Nutr Metab. 2008;52 Suppl 1:62-71.

13. Novakovic R, Cavelaars AE, Bekkering GE, Roman-Viñas B, Ngo J, Gurinović $\mathrm{M}$, et al. Micronutrient intake and status in Central and Eastern Europe compared with other European countries, results from the EURRECA network. Public Health Nutr. 2013;16:824-40.

14. Winkler $G$, Brasche $S$, Doring A, Heinrich J. Dietary intake of middle-aged men from an East and a West German city after the German reunification: do differences still exist? Eur J Clin Nutr. 1998;52:98-103.

15. Vandenbroucke JP, von Elm E, Altman DG, Gøtzsche PC, Mulrow CD, Pocock $\mathrm{SJ}$, et al. Strengthening the Reporting of Observational Studies in Epidemiology (STROBE): explanation and elaboration. Epidemiology. 2007;18:805-35.

16. Agudo A, Slimani N, Ocke MC, Naska A, Miller AB, Kroke A, et al. Consumption of vegetables, fruit and other plant foods in the European Prospective Investigation into Cancer and Nutrition (EPIC) cohorts from 10 European countries. Public Health Nutr. 2002;5:1179-96.

17. Trichopoulou A, Naska A, Costacou T. Disparities in food habits across Europe. Proc Nutr Soc. 2002;61:553-8.

18. Food and Agriculture Organization of the United Nations (2014): FAOSTAT. http://faostat3.fao.org/faostat-gateway/go/to/home/E

19. Peasey A, Bobak M, Kubinova R, Malyutina S, Pajak A, Tamosiunas A, et al. Determinants of cardiovascular disease and other non-communicable diseases in Central and Eastern Europe: rationale and design of the HAPIEE study. BMC Public Health. 2006;6:255. 
20. Powles JW, Day NE, Sanz MA, Bingham SA. Protective foods in winter and spring: a key to lower vascular mortality? Lancet. 1996;348:898-9.

21. Capita R, Alonso-Calleja C. Differences in reported winter and summer dietary intakes in young adults in Spain. Int J Food Sci Nutr. 2005;56:431-43.

22. Kromhout D, Keys A, Aravanis C, Buzina R, Fidanza F, Giampaoli S, et al. Food consumption patterns in the 1960s in seven countries. Am J Clin Nutr. 1989;49:889-94.

23. Schroll K, Carbajal A, Decarli B, Martins I, Grunenberger F, Blauw YH, et al. Food patterns of elderly Europeans SENECA Investigators. Eur J Clin Nutr. 1996:50 Suppl 2:S86-100.

24. Karamanos B, Thanopoulou A, Angelico F, Assaad-Khalil S, Barbato A, Del Ben $\mathrm{M}$, et al. Nutritional habits in the Mediterranean Basin. The macronutrient composition of diet and its relation with the traditional Mediterranean diet. Multi-centre study of the Mediterranean Group for the study of diabetes (MGSD). Eur J Clin Nutr. 2002;56:983-91.

25. Serra-Majem L, MacLean D, Ribas L, Brulé D, Sekula W, Prattala R, et al. Comparative analysis of nutrition data from national, household, and individual levels: Results from a WHO-CINDI collaborative project in Canada, Finland, Poland, and Spain. J Epidemiol Community Health. 2003;57:74-80.

26. Petkeviciene J, Simila M, Becker W, Kriaucioniene V, Valsta LM. Validity and reproducibility of the NORBAGREEN food frequency questionnaire. Eur J Clin Nutr. 2009;63:141-9.

27. Bobak M, Brunner E, Miller NJ, Skodová Z, Marmot M. Could antioxidants play a role in high rates of coronary heart disease in the Czech Republic? Eur J Clin Nutr. 1998:52:632-6.

28. Al-Delaimy WK, van Kappel AL, Ferrari P, Slimani N, Steghens JP, Bingham S, et al. Plasma levels of six carotenoids in nine European countries: report from the European Prospective Investigation into Cancer and Nutrition (EPIC). Public Health Nutr. 2004;7:713-22.

29. Kardinaal AFM, Kok FJ, Ringstad J, Gomez-Aracena J, Mazaev VP, Kohlmeier $L$, et al. Antioxidants in Adipose-Tissue and Risk of Myocardial-Infarction the Euramic Study. Lancet. 1993;342:1379-84.

30. Su LC, Bui M, Kardinaal A, Gomez-Aracena J, Martin-Moreno J, Martin B, et al. Differences between plasma and adipose tissue biomarkers of carotenoids and tocopherols. Cancer Epidemiol Biomarkers Prev. 1998;7:1043-8.

31. Winkler G, Holtz H, Doring A. Comparison of food intakes of selected populations in former East and West Germany: Results from the MONICA projects Erfurt and Augsburg. Ann Nutr Metab. 1992;36:219-34.

32. Lixandru D, Mohora M, Coman A, Stoian I, van Gils C, Aerts P, et al. Diet and paraoxonase 1 Enzymatic activity in diabetic foot patients from Romania and Belgium: Favorable association of high flavonoid dietary intake with arylesterase activity. Ann Nutr Metab. 2010;56:294-301.

33. Paalanen $L$, Prattala R, Palosuo H, Laatikainen T. Socio-economic differences in the consumption of vegetables, fruit and berries in Russian and Finnish Karelia: 1992-2007. Eur J Public Health. 2011;21:35-42.

34. Crispim SP, Geelen A, Souverein OW, Hulshof PJ, Ruprich J, Dofkova M, et al. Biomarker-based evaluation of two 24-h recalls for comparing usual fish, fruit and vegetable intakes across European centers in the EFCOVAL Study. Eur J Clin Nutr. 2011;65 Suppl 1:S38-47.

35. El Ansari W, Stock C, Mikolajczyk RT. Relationships between food consumption and living arrangements among university students in four European countries - a cross-sectional study. Nutr J. 2012;11:28.

36. Wardle J, Steptoe A, Bellisle F, Davou B, Reschke K, Lappalainen R, et al. Healthy dietary practices among European students. Health Psychol. 1997;16:443-50.

37. Prattala R, Paalanen L, Grinberga D, Helasoja V, Kasmel A, Petkeviciene J. Gender differences in the consumption of meat, fruit and vegetables are similar in Finland and the Baltic countries. Eur J Public Health. 2007;17:520-5

38. Prattala R, Hakala S, Roskam AJR, Roos E, Helmert U, Klumbiene J, et al. Association between educational level and vegetable use in nine European countries. Public Health Nutr. 2009;12:2174-82.

39. Hall JN, Moore S, Harper SB, Lynch JW. Global variability in fruit and vegetable consumption. Am J Prev Med. 2009;36:402-9.

40. European Commission (2013): European Health Interview Survey. Eurostat. http://ec.europa.eu/eurostat/data/database

41. Burisch J, Pedersen N, Cukovic-Cavka S, Turk N, Kaimakliotis I, Duricova D, et al. Environmental factors in a population-based inception cohort of inflammatory bowel disease patients in Europe-an ECCO-EpiCom study. J Crohns Colitis. 2014:8:607-16.
42. Kristenson $M$, Zieden B, Kucinskiene $Z$, Elinder LS, Bergdahl B, Elwing B, et al. Antioxidant state and mortality from coronary heart disease in Lithuanian and Swedish men: concomitant cross sectional study of men aged 50. BMJ. 1997:314:629-33.

43. Bobak M, Hense HW, Kark J, Kuch B, Vojtisek P, Sinnreich R, et al. An ecological study of determinants of coronary heart disease rates: a comparison of Czech, Bavarian and Israeli men. Int J Epidemiol. 1999;28:437-44.

44. Miere D, Filip L, Indrei LL, Soriano JM, Molto JC, Manes J. Nutritional assessment of the students from two European university centers [Romanian] Evaluarea nutritionala a studentilor din doua centre universitare Europene. Rev Med Chir Soc Med Nat lasi. 2007;111:270-5.

45. Woodside JV, Young IS, Gilchrist SE, Vioque J, Chakravarthy U, de Jong PT, et al. Factors associated with serum/plasma concentrations of vitamins A, C, E and carotenoids in older people throughout Europe: the EUREYE study. Eur J Nutr. 2013;52:1493-501.

46. National and Kapodistrian University of Athens (2005): Data Food Networking (DAFNE) databank. http://www.nut.uoa.gr/dafnesoftweb/

47. Jenab M, Salvini S, van Gils CH, Brustad M, Shakya-Shrestha S, Buijsse B, et al. Dietary intakes of retinol, beta-carotene, vitamin $D$ and vitamin $E$ in the European Prospective Investigation into Cancer and Nutrition cohort. Eur J Clin Nutr. 2009;63 Suppl 4:S150-78.

48. European Food Safety Authority's Advisory Forum on the Pan-European Food Consumption Survey (2010): What's on the menu in Europe? - a Pan-European Food Consumption Survey (EU Menu). Seville. http:// www.efsa.europa.eu/en/eumenu/docs/eumenudeclarationen.pdf

49. Dauchet $L$, Amouyel P, Dallongeville J. Fruits, vegetables and coronary heart disease. Nat Rev Cardiol. 2009;6:599-608.

50. Boeing $\mathrm{H}$, Bechthold A, Bub A, Ellinger S, Haller D, Kroke A, et al. Critical review: vegetables and fruit in the prevention of chronic diseases. Eur J Nutr. 2012:51:637-63

\section{Submit your next manuscript to BioMed Central and take full advantage of:}

- Convenient online submission

- Thorough peer review

- No space constraints or color figure charges

- Immediate publication on acceptance

- Inclusion in PubMed, CAS, Scopus and Google Scholar

- Research which is freely available for redistribution 